\title{
Editorial
}

\section{Unusual presentations in patients with systemic lupus erythematosus: a result of disease activity or something else?}

Anisur Rahman

Department of Rheumatology, University College London, London, UK, anisur.rahman@ucl.ac.uk

Standfirst

Systemic lupus erythematosus can affect any organ or tissue, but skin manifestations, joint pain and fatigue are the most common symptoms. Two case reports in this issue describe patients with systemic lupus erythematosus who suffered severe internal organ manifestations.

\section{Introduction}

Systemic lupus erythematosus is a systemic autoimmune disease with a prevalence of 97 per 100000 in the UK (Rees et al, 2016). It can affect any organ or tissue in the body, but skin manifestations, joint pain and fatigue are the most common symptoms occurring in almost all patients at some stage of the disease. In deciding whether new symptoms, or a flare of existing symptoms, are caused by systemic lupus erythematosus disease activity, blood tests such as erythrocyte sedimentation rate, titre of anti-double-stranded-DNA antibodies (anti-dsDNA) and levels of complement components $\mathrm{C} 3$ and $\mathrm{C} 4$ are helpful.

In this issue of the journal, two groups (Tan et al and Deoraj et al) describe scenarios where patients with systemic lupus erythematosus suffered severe internal organ manifestations but it was difficult to determine whether or not these were caused by systemic lupus erythematosus itself.

\section{Neuropsychiatric systemic lupus erythematosus}

Tan et al (https://doi.org/10.12968/hmed.2020.0049) describe a case in which a patient with known systemic lupus erythematosus presented with neurological features including headache, a seizure and expressive dysphasia. Multiple investigations did not suggest high systemic lupus erythematosus disease activity but were very important in excluding other causes of the clinical picture, such as thrombosis or infection. Ultimately, treatment with intravenous methylprednisolone and cyclophosphamide was successful dramatically improving the patient's symptoms.

The American College of Rheumatology defined multiple different syndromes that could arise from neuropsychiatric systemic lupus erythematosus (Liang et al, 1999), but these include symptoms such as headache and mood disorders that are common in the general population. Therefore, in most patients with these common symptoms, systemic lupus erythematosus is not the underlying cause. In an inception cohort study, the Systemic Lupus International Collaborative Clinics (SLICC) group described 843 neuropsychiatric events in a population of 1206 patients with newly diagnosed systemic lupus erythematosus followed for a mean of 1.9 years (Hanly et al, 2010). Although $40 \%$ of all patients developed a neuropsychiatric event during this period, the majority of those events were not caused by active systemic lupus erythematosus (Hanly et al, 2010).

The main clues to determining whether a patient has neuropsychiatric systemic lupus erythematosus are whether there is also activity of the disease in other organs, eg rash, and whether there is elevated anti-dsDNA levels and/or erythrocyte sedimentation rate and/or reduced complement levels. Computed tomography scanning is useful to exclude haemorrhage or infarct but 
magnetic resonance imaging is preferred to look for changes suggestive of cerebral systemic lupus erythematosus. Nevertheless, no specific pattern on any form of imaging is diagnostic of neuropsychiatric systemic lupus erythematosus (Sarbu et al, 2015). A number of novel serological and imaging modalities have been suggested for diagnosis and management of this condition and have been reviewed elsewhere (Clark et al, 2017). Currently, however, clinicians may still be confronted with cases like this one in which investigations are normal or near-normal and the diagnosis of neuropsychiatric systemic lupus erythematosus is based on the balance of clinical probabilities and response to treatment.

\section{Cardiac manifestations of systemic lupus erythematosus}

The second case, reported by Deoraj et al (https://doi.org/10.12968/hmed.2020.0053), describes a case of sudden-onset biventricular failure in a patient with treated lupus nephritis. In this case, the authors rightly stress the fact that systemic lupus erythematosus might well not have been the underlying cause of the patient's deterioration and list multiple factors that might have contributed to cardiomyopathy. They particularly note a possible role for medications, and it is interesting that the patient developed heart failure shortly after a course of B-cell depletion. On the other hand, the patient had raised anti-dsDNA and reduced C3 levels at the time of the event, and improved with corticosteroids in combination with anti-heart failure treatment. The latter point is important; where the cause of a deterioration in a patient with systemic lupus erythematosus is unclear it remains important to provide supportive treatment for the end-organ effects, eg anticonvulsants for seizures.

Although systemic lupus erythematosus is associated with increased risk of developing coronary disease (Manzi et al, 1997), the cardiac magnetic resonance scan in this patient was not suggestive of ischaemic cardiomyopathy. It did not show the pattern on late gadolinium enhancement that has been reported in other cardiac magnetic resonance studies in systemic lupus erythematosus (O Neill et al, 2009).

\section{Conclusions}

These cases illustrate the difficulty of distinguishing active systemic lupus erythematosus from other causes such as medication side effects in patients with systemic lupus erythematosus who experience a sudden deterioration. In the absence of definitive results from investigations, clinical acumen and appropriate use of supportive therapies are very important.

Key points

1) Neuropsychiatric SLE can present with a wide variety of symptoms, and these range from very common symptoms such as headache to severe manifestations such as seizures or psychosis.

2) It is important to recognize that mild symptoms such as headache and mood disorder in a patient with SLE are probably not due to SLE itself.

3) In deciding whether neuropsychiatric symptoms are due to SLE, it is important to take into account whether other organs are active and the levels of blood markers such as anti-dsDNA and complement levels.

4) When patients with SLE develop heart failure, there are various possible causes including medication side-effects.

5) Remember to provide treatment for the end-organ effects of flares of SLE, such as anti-convulsants for seizures and heart failure medication where required. 


\section{References}

Clark KE, Clark CN, Rahman A. A critical analysis of the tools to evaluate neuropsychiatric lupus. Lupus. 2017;26(5):504-509. https://doi.org/10.1177/0961203317690242

Hanly JG, Urowitz MB, Su L et al. Prospective analysis of neuropsychiatric events in an international disease inception cohort of patients with systemic lupus erythematosus. Ann Rheum Dis. 2010;69(3):529-535. https://doi.org/ https://doi.org/10.1136/ard.2008.106351

Liang MH, Corzillius M, Bae S et al. The American College of Rheumatology nomenclature and case definitions for neuropsychiatric lupus syndromes. Arthritis Rheum. 1999;42(4):599-608. https://doi.org/10.1002/1529-0131(199904)42:4<599::AID-ANR2>3.0.CO;2-F

Manzi S, Meilahn EN, Rairie JE et al. Age-specific incidence rates of myocardial infarction and angina in women with systemic lupus erythematosus: comparison with the Framingham Study. Am J Epidemiol. 1997;145(5):408-415. https://doi.org/10.1093/oxfordjournals.aje.a009122

O Neill SG, Woldman S, Bailliard F et al. Cardiac magnetic resonance imaging in patients with systemic lupus erythematosus. Ann Rheum Dis. 2009;68(9):1478-1481.

https://doi.org/10.1136/ard.2008.098053

Rees F, Doherty M, Grainge M et al. The incidence and prevalence of systemic lupus erythematosus in the UK, 1999-2012. Ann Rheum Dis. 2016;75(1):136-141. https://doi.org/10.1136/annrheumdis2014-206334

Sarbu N, Alobeidi F, Toledano P et al. Brain abnormalities in newly diagnosed neuropsychiatric lupus: systematic MRI approach and correlation with clinical and laboratory data in a large multicenter cohort. Autoimmun Rev. 2015;14(2):153-159.

https://doi.org/10.1016/j.autrev.2014.11.001 